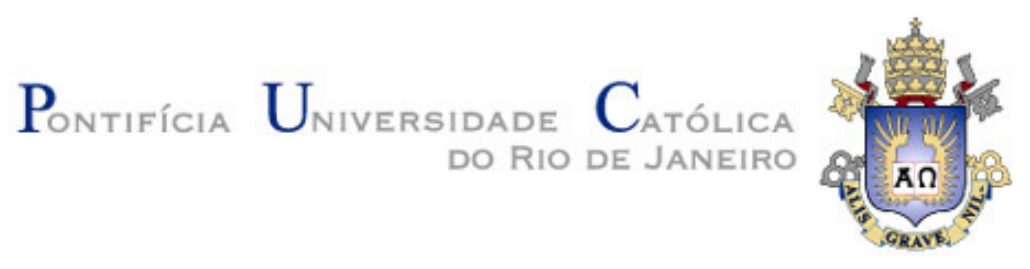

Carmen Pilar Castro Barrientos

Contribuições à Avaliação Metrológica de Esterilizadores por Vapor Saturado

Dissertação de Mestrado

Dissertação apresentada ao Programa de PósGraduação em Metrologia da PUC-Rio como requisito parcial para obtenção do titulo de mestre em Metrologia. Área de concentração: Metrologia para Qualidade e Inovação.

Orientador: Prof. Elisabeth Costa Monteiro Co-orientador: Prof. Carlos Roberto Hall Barbosa 


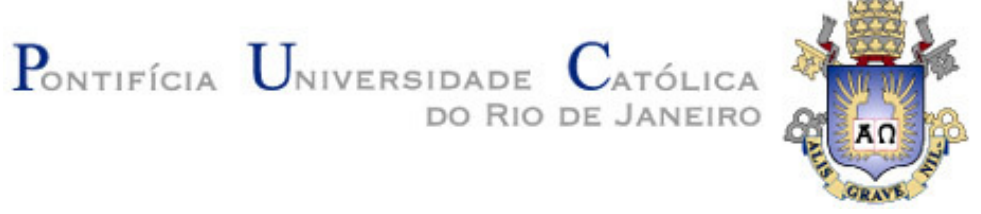

Carmen Pilar Castro Barrientos

\title{
Contribuições à Avaliação Metrológica de Esterilizadores \\ por Vapor Saturado
}

\begin{abstract}
Dissertação apresentada como requisito parcial para obtenção do grau de Mestre pelo Programa de PósGraduação em Metrologia do Centro Técnico da PUC-Rio. Aprovada pela Comissão Examinadora e homologada pela Coordenação Setorial de Pós-Graduação,formalizado pelas respectivas assinaturas.
\end{abstract}

Comissão Examinadora :

Prof. Elisabeth Costa Monteiro

Orientador

Programa de Pós-Graduação em Metrologia (PósMQI/PUC-Rio)

Prof. Carlos Roberto Hall Barbosa

Co-Orientador

Programa de Pós-Graduação em Metrologia (PósMQI/PUC-Rio)

Prof. Maurício Nogueira Frota

Programa de Pós-Graduação em Metrologia (PósMQl/PUC-Rio)

Prof. Reinaldo Castro Souza

Departamento de Engenheria Elétrica

Pontifícia Universidade Católica do Rio de Janeiro

Coordenação Setorial de Pós-Graduação:

Prof. José Eugenio Leal

Coordenador Setorial do Centro Técnico Científico - PUC-Rio

Rio de Janeiro, 27 de abril de 2012 
Todos os direitos reservados. É proibida a reprodução total ou parcial do trabalho sem autorização da universidade, da autora e do orientador.

\section{Carmen Pilar Castro Barrientos}

Formado em Ciencias Fisicas pela Universidad Nacional Federico Villarreal,UNFV (Lima, Perú) em 2006. Cursou mestrado na PUC-Rio em 2009, especializando-se em Metrologia Qualidade e Inovação.

Ficha Catalográfica

Castro Barrientos, Carmen Pilar

Contribuições à avaliação metrológica de esterilizadores por vapor saturado / Carmen Pilar Castro Barrientos ; orientador: Elisabeth Costa Monteiro ; coorientador: Carlos Roberto Hall Barbosa. - 2012.

142 f. : il. (color.) ; $30 \mathrm{~cm}$

Dissertação (mestrado)-Pontifícia Universidade Católica do Rio de Janeiro, Programa de Pós-Graduação em Metrologia para a Qualidade e Inovação, 2012.

Inclui bibliografia

1. Metrologia - Teses. 2. Esterilização. 3. Infecção hospitalar. 4. Controle de infecção. 5. Microbactéria. 6. Autoclave. I. Monteiro, Elisabeth Costa. II. Barbosa, Carlos Roberto Hall. III. Pontifícia Universidade Católica do Rio de Janeiro. Programa de Pós-Graduação em Metrologia para a Qualidade e Inovação. IV. Título. 


\section{Agradecimentos}

A Deus, pelas forças que me permite continuar.

À valiosa colaboração da orientadora e professora $\operatorname{Dr}^{\mathrm{a}}$. Elisabeth Costa Monteiro, pesquisadora assídua, que muitas vezes com muita determinação me encorajou a prosseguir nesta batalha árdua e ao meu co-orientador Professor Carlos Hall.

À Engeclinic Serviços Ltda e PUC-Rio, pelos auxílios concedidos, sem os quais este trabalho não poderia ter sido realizado.

A todos os professores, colegas e funcionários do Programa de Pós-Graduação em Metrologia do centro Técnico Científico da PUC-Rio pelos ensinamentos e momentos compartilhados e conselhos a nível acadêmico.

Aos professores que participaram da Comissão examinadora

A todos os amigos e familiares que, de uma forma ou de outra, me estimularam ou me ajudaram neste crescimento cultural, em especial à minha mãe Victoria $\mathrm{P}$. Barrientos Baca. 


\section{Resumo}

Castro Barrientos, Carmen Pilar; Costa Monteiro, Elisabeth; Hall Barbosa, Carlos Roberto. Contribuições à Avaliação Metrológica de Esterilizadores por Vapor Saturado. Rio de Janeiro, 2012. 142 p. Dissertação de Mestrado - Departamento de Metrologia (Área de concentração: Metrologia para Qualidade e Inovação), Pontifícia Universidade Católica do Rio de Janeiro.

No Brasil, as Infecções Hospitalares se constituem na principal causa de mortalidade de pacientes internados, gerando grandes prejuízos financeiros. Em 2008, a ANVISA publicou alerta de "emergência epidemiológica" devido à ocorrência de infecções por micobactérias de crescimento rápido em todas as regiões do país, infecções fortemente relacionadas a falhas nos processos de limpeza, desinfecção e esterilização de produtos médicos. A validação é uma ferramenta da metrologia que possibilita estabelecer se o processo de esterilização é executado de modo eficaz e reprodutível. Na norma ISO/TS 17665-2:2009 sobre validação da esterilização de produtos para saúde, se destacam recomendações de revalidações periódicas, distribuição espacial de sensores térmicos, critérios de aceitação quanto à temperatura calculada a partir da pressão medida na câmara e estimativa de incerteza de medição. Com base nos requisitos, recomendações, orientações e exigências descritas em documentos nacionais e internacionais, no presente trabalho se desenvolvem sistema e procedimento para avaliação da confiabilidade metrológica de esterilizadores por vapor saturado. Com o sistema foram avaliadas quatro autoclaves gravitacionais em uso hospitalar e industrial. Os resultados reforçam a importância de aspectos como: revalidações, condições ambientais e impacto da estabilidade temporal e uniformidade espacial na incerteza global das câmaras. Em todas as autoclaves avaliadas o processo se realizou por vapor superaquecido, que é menos eficiente e não-conforme à ISO 17665. As limitações identificadas corroboram com os novos requisitos da ANVISA (RDC 15:2012), tanto com relação à exigência de avaliações periódicas de esterilizadores, quanto à proibição do uso de autoclaves gravitacionais com capacidade superior a $100 \mathrm{~L}$.

\section{Palavras-chave}

Esterilização; Infecção hospitalar; controle de infecção; micobactéria; Autoclave. 


\section{Abstract}

Carmen Barrientos, Pilar Castro; Costa Monteiro, Elisabeth (Advisor); Hall Barbosa, Carlos Roberto (Co-Advisor). Contributions on the Metrological Evaluation of Saturated Steam Sterilizers. Rio de Janeiro, 2012. 142p. MSc. Dissertation - Departamento de Metrologia (Área de concentração: Metrologia para Qualidade e Inovação), Pontifícia Universidade Católica do Rio de Janeiro.

In Brazil, the Hospital Infections are the main cause of mortality during hospitalization, generating huge financial losses. In 2008, ANVISA issued a warning of "epidemiological emergency" due to the occurrence of infections by rapidly growing mycobacteria in all regions of the country, strongly related to failures in the processes of cleaning, disinfection and sterilization of medical products. Validation is a metrological tool that allows establishing whether the sterilization process developed is running efficiently and with reproducibility. ISO/TS 17665-2:2009, on validation of sterilization products for health, highlights the need of periodic revalidations, measurements of temperature with sensors distributed throughout the chamber volume, specifies acceptance criteria based on measurements of pressure in the internal volume of the chamber (theoretical temperature) and introduces the measurement uncertainty requirement. In this work, based on the requirements and recommendations outlined in national and international documents, a system and procedure are developed for the metrological reliability evaluation of saturated steam

sterilizers. With the developed system, four gravitational autoclaves were evaluated. The results reinforce the importance of revalidation, environmental conditions, and the contribution of temporal stability and spatial uniformity in the overall uncertainty of the cameras. All the autoclave process was carried out by means of superheated steam, which is less efficient and non-conform to ISO 17665. The limitations identified corroborate with the recently published requirements of RDC ANVISA 15:2012, concerning both the need of periodic evaluations of sterilizers and the prohibition of the use of gravitational autoclaves with capacity exceeding $100 \mathrm{~L}$.

\section{Keywords}

Sterilization; hospital Infection; infection control; mycobacteria; Autoclave. 


\section{Sumário}

1 Introdução 17

1.1. Organização da Dissertação 20

2 Esterilização $\quad 21$

2.1. Métodos de Esterilização 21

2.1.1. Métodos Químicos $\quad 21$

2.1.1.1. Métodos Químicos líquidos $\quad 21$

2.1.1.2. Métodos Químicos gasosos 23

2.1.2. Métodos Físico-Químicos 23

2.1.3. Métodos Físicos $\quad 24$

2.1.3.1. Esterilização térmica por calor seco 24

2.1.3.2. Esterilização térmica por vapor saturado 25

2.2. Esterilização por vapor saturado 26

$\begin{array}{ll}\text { 2.2.1. Vapor saturado } & 27\end{array}$

2.2.2. Monitorização Química e Biológica 30

3 Confiabilidade metrológica em esterilizadores por vapor 32

3.1. Estrutura metrológica 32

3.1.1. Contexto internacional 33

3.1.1.1. BIPM 33

3.1.1.2. OIML 34

3.1.1.3. ILAC 35

3.1.1.4. WHO 35

3.1.1.5. IAPWS 36

3.1.1.6. ISO 36

3.1.1.7. IEC 40

3.1.1.8. CEN 41

3.1.1.9. Outros Institutos de normalização 42

3.1.2. Contexto Nacional 43

3.1.2.1. INMETRO 43 
3.1.2.2. ANVISA 46

$\begin{array}{ll}\text { 3.1.2.3. ABNT } & 48\end{array}$

3.2. Validação e Estimativa da Incerteza de Medição 50

3.2.1. Validação 50

3.2.1.1. Qualificação de instalação 50

3.2.1.2. Qualificação operacional 51

3.2.1.3. Qualificação de desempenho 51

3.2.2. Incerteza de medição 52

4 Materiais e Métodos $\quad 56$

4.1. Sistema de Medição $\quad 59$

4.2. Validação do processo de esterilização 67

4.3. Estimativa das incertezas no esterilizador 72

5 Resultados 76

5.1. Condições ambientais das medições de validação 76

5.2. Distribuição espacial da temperatura e estabilidade térmica temporal dos esterilizadores $\quad 78$

5.2.1. Esterilizador $\mathrm{E}_{1} \quad 78$

5.2.1.1. Ciclo de esterilização sem carga do esterilizador $E_{1} \quad 78$

5.2.1.2. Ciclo de esterilização com carga do esterilizador $E_{1} \quad 91$

5.2.2. Esterilizador $E_{2} \quad 105$

5.2.3. Esterilizador $E_{3} \quad 118$

$\begin{array}{ll}\text { 5.2.4. Esterilizador } \mathrm{E}_{4} & 124\end{array}$

6 Discussão 131

7 Conclusão 135

8 Referências Bibliográficas 137 


\section{Lista de figuras}

Figura 1 - Representação esquemática da superfície termodinâmica e projeções nos planos (ANGELO E. e SIMÕES M.J.R., 2011).............28

Figura 2 - Relação pressão $x$ temperatura

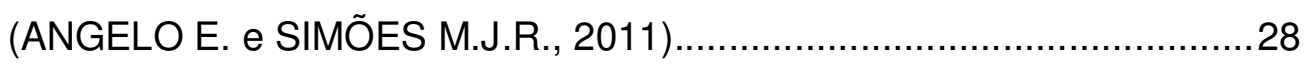

Figura 3 - Representação da distribução Normal .....................................54

Figura 4 - Representação da distribução retangular ...............................54

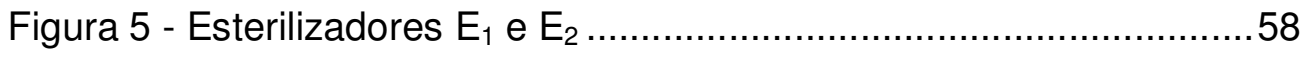

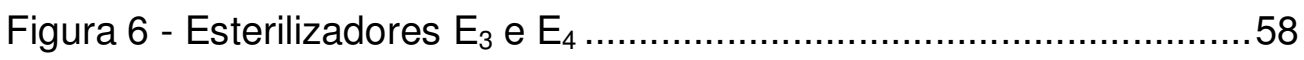

Figura 7 - Diagrama esquemático de um sistema de medição...................59

Figura 8 - Sistema de medição de temperatura …………………..........60

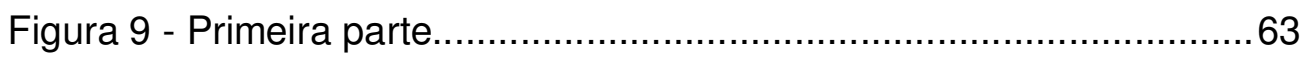

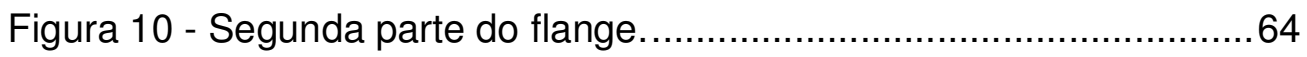

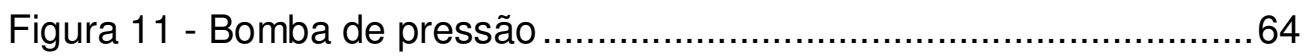

Figura 12 - Indicador químico descartável classe II................................65

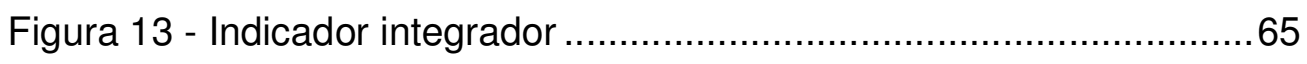

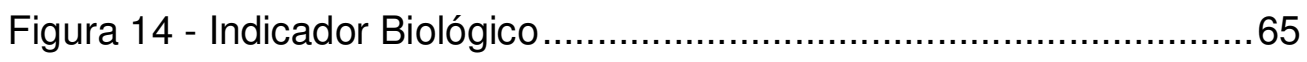

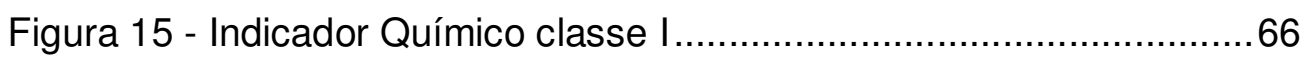

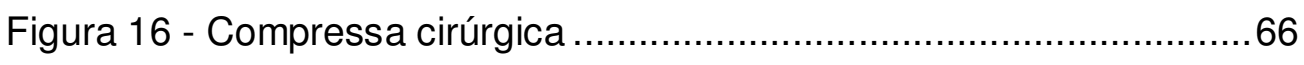

Figura 17 - Distribuição dos transdutores de temperatura na

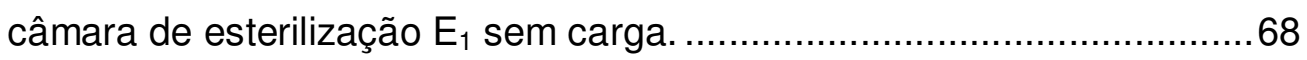

Figura 18 - Câmara com carga do esterilização $E_{1}$.................................69

Figura 19 - Câmara com carga do esterilização $E_{2}$.................................. 69

Figura 20 - Suportes para os produtos de saúde a serem esterilizados ..70

Figura 21 - Distribuição dos transdutores nos esterilizadores E1 e E2 ...70

Figura 22 - Distribuição dos transdutores no esterilizador $\mathrm{E}_{3} \ldots \ldots \ldots \ldots \ldots \ldots . . . . .71$

Figura 23 - Distribuição dos transdutores no esterilizador $\mathrm{E}_{4} \ldots \ldots \ldots \ldots \ldots \ldots \ldots . . . . . . .71$

Figura 24 - Estabilidade Térmica Temporal da câmara $E_{1}$

sem carga no primeiro ciclo.

Figura 25 - Temperatura teórica no primeiro ciclo da câmara 
$\mathrm{E}_{1}$ sem carga.

Figura 26 - Estabilidade Térmica Temporal da câmara

$\mathrm{E}_{1}$ sem carga no segundo ciclo.

Figura 27 - Estabilidade Térmica Temporal da câmara

$\mathrm{E}_{1}$ sem carga no terceiro ciclo.

Figura 28 - Médias das temperaturas e suas incertezas obtidas

dos transdutores no equipamento $E_{1}$ sem carga no primeiro ciclo.

Figura 29 - Média das temperaturas e suas incertezas obtidas

dos transdutores no equipamento $E_{1}$ sem carga no segundo ciclo.

Figura 30 - Média das temperaturas e suas incertezas obtidas

dos transdutores no equipamento $E_{1}$ sem carga no terceiro ciclo.

Figura 31 - Localização e distribuição espacial dos transdutores nas câmaras $\left(E_{1}\right.$ e $\left.E_{2}\right)$ e indicador químico.

Figura 32 - Temperatura em função da pressão no interior de $E_{1}$ sem carga, para o primeiro ciclo ( $T_{P}$ calculado com base no IAPWS,97).

Figura 33 - Temperatura em função da pressão no interior de $E_{1}$ sem carga, para o segundo ciclo ( $T_{\mathrm{P}}$ calculado com base no IAPWS,97) 90

Figura 34 - Temperatura em função da pressão no interior de $E_{1}$ sem carga, para o terceiro ciclo ( $T_{P}$ calculado com base no IAPWS,97) 90

Figura 35 - Estabilidade Térmica Temporal da câmara $E_{1}$ com carga no primeiro ciclo.

Figura 36 - Estabilidade Térmica Temporal da câmara $E_{1}$ com carga no segundo ciclo.

Figura 37 - Estabilidade Térmica Temporal da câmara $E_{1}$ com carga no terceiro ciclo. .96

Figura 38 - Média das temperaturas e suas incertezas obtidas em cada transdutor no equipamento $E_{1}$ com carga no primeiro ciclo.

Figura 39 - Média das temperaturas e suas incertezas obtidas em cada transdutor no equipamento $E_{1}$ com carga no segundo ciclo.....98 Figura 40 - Média das temperaturas e suas incertezas obtidas em cada transdutor no equipamento $E_{1}$ com carga no terceiro ciclo.......98 
Figura 41 - Localizações dos desafios Biológicos .99

Figura 42 - Indicador biológico depois da esterilização.

Figura 43 - Temperatura em função da pressão no interior de $E_{1}$ com carga, para o primeiro ciclo ( $T_{P}$ calculado com base no IAPWS,97) 103

Figura 44 - Temperatura em função da pressão no interior de $E_{1}$ com carga, para o segundo ciclo ( $T_{P}$ calculado com base no IAPWS,97). 104

Figura 45 - Temperatura em função da pressão no interior de $E_{1}$ com carga, para o terceiro ciclo ( $T_{P}$ calculado com base no IAPWS,97). 104

Figura 47 - Estabilidade Térmica Temporal da câmara $E_{2}$ com carga no primeiro ciclo. 109

Figura 48 - Estabilidade Térmica Temporal da câmara $E_{2}$ com carga no segundo ciclo.

Figura 49 - Estabilidade Térmica Temporal da câmara $E_{2}$ com carga no terceiro ciclo. 110

Figura 50 - Média das temperaturas e suas incertezas obtidas em cada transdutor no equipamento $E_{2}$ com carga no primeiro ciclo....111 Figura 51 - Média das temperaturas e suas incertezas obtidas em cada transdutor no equipamento $E_{2}$ com carga no segundo ciclo...112 Figura 52 - Média das temperaturas e suas incertezas obtidas em cada transdutor no equipamento $\mathrm{E}_{2}$ com carga no terceiro ciclo.....112 Figura 53 - Localizações dos desafios Biológicos (cor verde) e as temperaturas mais quentes (cor vermelho) e mais fria (azul)

Figura 54 - Temperatura em função da pressão no interior de $\mathrm{E}_{2}$ sem carga, para o primeiro ciclo ( $T_{P}$ calculado com base no IAPWS,97) 116

Figura 55 - Temperatura em função da pressão no interior de $E_{2}$ sem carga, para o segundo ciclo ( $T_{P}$ calculado com base no IAPWS,97).

Figura 56 - Temperatura em função da pressão no interior de $E_{2}$ sem carga, para o terceiro ciclo ( $T_{\mathrm{P}}$ calculado com base 
no IAPWS,97)

Figura 57 - Estabilidade Térmica Temporal da câmara $E_{3}$ sem carga...120

Figura 58 - Média das temperaturas e suas incertezas obtidas

em cada transdutor no equipamento $E_{3}$

Figura 59 - Distribuição espacial dos transdutores no volume útil

de $\mathrm{E}_{3}$.

Figura 60 - Temperatura em função da pressão no interior de

$E_{3}$ sem carga, para o terceiro ciclo $\left(T_{P}\right.$ calculado com base no IAPWS,97).

Figura 61 - Estabilidade Térmica Temporal da câmara do $E_{4}$

sem carga.

Figura 62 - Média das temperaturas e suas incertezas obtidas em cada transdutor no equipamento $\mathrm{E}_{4}$

Figura 63 - Distribuição espacial dos transdutores no volume útil de $\mathrm{E}_{4}$ 128

Figura 64 - Temperatura em função da pressão no interior de $E_{4}$ sem carga, para o terceiro ciclo ( $T_{p}$ calculado com base no IAPWS,97). 130 


\section{Lista de tabelas}

Tabela 1 - Relação de valores de temperatura e tempo de exposição necessários para esterilização por calor seco (OPS, 2008)...25

Tabela 2 - Relação de valores de temperatura e tempo de exposição necessários para esterilização por vapor saturado (ISO/TS 17665-2,2009).

Tabela 3 - Valores de temperatura e pressão do vapor saturado (ISO 17665 - 2009) .30

Tabela 4 - Classificação dos indicadores Químicos. 31

Tabela 5 - Grupos de Trabalho do Comitê Técnico ISO/TC 198 .38

Tabela 6 - Algumas normas ISO relacionadas com esterilização. 39

Tabela 7 - Publicações IEC relacionadas com os esterilizadores 40

Tabela 8 - Normas EN relacionadas com esterilização de produtos.

Tabela 9 - Documentos publicados pela CGCRE relacionados

a câmaras térmicas.

Tabela 10 - Publicações relacionadas ao processo de esterilização. 46

Tabela 11 - Alguns dos requisitos publicados pela RDC 15 de 15/03/2012.

Tabela 12 - Publicações da ABNT relacionadas a processos de esterilização

Tabela 13 - Especificações dos esterilizadores avaliados .56

Tabela 14 - Características dos quatro equipamentos $\left(E_{1}, E_{2}, E_{3}, E_{4}\right) \ldots .57$

Tabela 15 - Composição dos termopares utililzados no sistema de medição.

Tabela 16 - Características das medições realizadas em cada um dos quatro esterilizadores a vapor avaliados.

Tabela 17 - Condições ambientais nos equipamentos avaliados.

Tabela 18 - Resultados no primeiro ciclo de ensaio termométrico realizado no esterilizador E1 sem carga. .79

Tabela 19 - Resultados no segundo ciclo de ensaio 
termométrico no esterilizador $\mathrm{E}_{1}$ sem carga.

Tabela 20 - Resultados do terceiro ciclo de ensaio termométrico

no esterilizador $E_{1}$ sem carga.

Tabela 21 - Balanço das incertezas na câmara sem carga de $E_{1}$

no primeiro ciclo.

Tabela 22 - Balanço das incertezas na câmara sem carga de $E_{1}$ no segundo ciclo

Tabela 23 - Balanço das incertezas na câmara sem carga de $E_{1}$

durante o terceiro.

Tabela 24 - Tabela de resultados no primeiro ciclo de ensaio

termométrico realizado no esterilizador $\mathrm{E}_{1}$ com carga

Tabela 25 - Tabela de resultados no segundo ciclo de ensaio termométrico realizado no esterilizador $E_{1}$ com carga.

Tabela 26 - Tabela de resultados no terceiro ciclo de ensaio

termométrico realizado no esterilizador $\mathrm{E}_{1}$ com carga

Tabela 27 - Balanço das incertezas na câmara $E_{1}$ com carga no

primeiro ciclo.

Tabela 28 - Balanço das incertezas na câmara E1 com carga no segundo ciclo.

Tabela 29 - Balanço das incertezas na câmara E1 com carga no terceiro ciclo

Tabela 30 - Balanço das incertezas na câmara $E_{1}$ (sem carga e com carga).

Tabela 31 - Tabela de resultados no primeiro ciclo com carga

realizado no esterilizador $\mathrm{E}_{2}$.

Tabela 32 - Tabela de resultados no segundo ciclo de ensaio

termométrico realizado no esterilizador $\mathrm{E}_{2}$ com carga.

Tabela 33 - Tabela de resultados no terceiro ciclo com carga

realizado no esterilizador $\mathrm{E}_{2}$

Tabela 34 - Balanço das incertezas do ciclo 1 na câmara $E_{2}$

com carga.

Tabela 35 - Balanço das incertezas do ciclo 2 na câmara $E_{2}$ com carga. 
Tabela 36 - Balanço das incertezas do ciclo 3 na câmara $E_{2}$

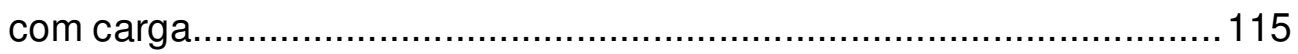

Tabela 37 - Balanço das incertezas na câmara $E_{2}$ para as medições com carga. 115

Tabela 38 - Tabela de resultados no ensaio termométrico realizado no esterilizador $E_{3}$ sem carga...........................................119

Tabela 39 - Balanço das incertezas na câmara do $E_{3}$ sem carga ...........122

Tabela 40 - Balanço das incertezas na câmara do $E_{3}$ sem carga...........123

Tabela 41- Resultados no esterilizador $\mathrm{E}_{4}$ sem carga .....................125

Tabela 42 - Balanço das incertezas na câmara E4 sem carga ..............128

Tabela 43 - Balanço das incertezas na câmara $E_{4}$ para as medições sem carga. 


\section{Lista de quadros}

Quadro 1- Grandezas de base e unidades de base do SI (BIPM,

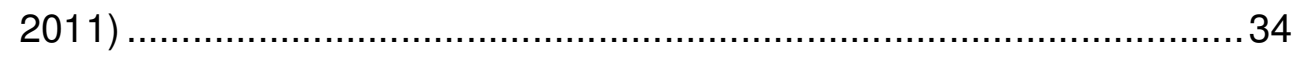

